\title{
Getting to grips with endoscopy - Learning endoscopic surgical skills induces bi- hemispheric plasticity of the grasping network
}

Karabanov, Anke Ninija; Irmen, Friederike; Madsen, Kristoffer Hougaard; Haagensen, Brian Numelin; Schulze, Svend; Bisgaard, Thue; Siebner, Hartwig Roman

\section{Published in:}

Neurolmage

Link to article, DOI:

10.1016/j.neuroimage.2018.12.030

Publication date:

2019

Document Version

Peer reviewed version

Link back to DTU Orbit

Citation (APA):

Karabanov, A. N., Irmen, F., Madsen, K. H., Haagensen, B. N., Schulze, S., Bisgaard, T., \& Siebner, H. R. (2019). Getting to grips with endoscopy - Learning endoscopic surgical skills induces bi-hemispheric plasticity of the grasping network. Neurolmage, 189, 32-44. https://doi.org/10.1016/j.neuroimage.2018.12.030

\section{General rights}

Copyright and moral rights for the publications made accessible in the public portal are retained by the authors and/or other copyright owners and it is a condition of accessing publications that users recognise and abide by the legal requirements associated with these rights.

- Users may download and print one copy of any publication from the public portal for the purpose of private study or research.

- You may not further distribute the material or use it for any profit-making activity or commercial gain

- You may freely distribute the URL identifying the publication in the public portal 


\section{Accepted Manuscript}

Getting to grips with endoscopy - Learning endoscopic surgical skills induces bihemispheric plasticity of the grasping network

Anke Ninija Karabanov, Friederike Irmen, Kristoffer Hougaard Madsen, Brian Numelin Haagensen, Svend Schulze, Thue Bisgaard, Hartwig Roman Siebner

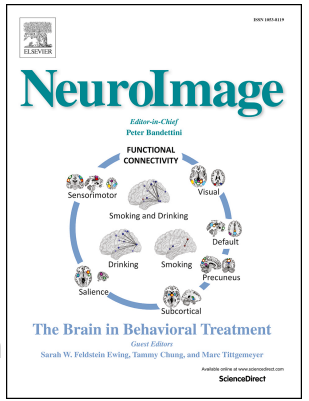

PII:

S1053-8119(18)32174-8

DOI: https://doi.org/10.1016/j.neuroimage.2018.12.030

Reference: $\quad$ YNIMG 15498

To appear in: Neurolmage

Received Date: 16 August 2018

Revised Date: 12 December 2018

Accepted Date: 14 December 2018

Please cite this article as: Karabanov, A.N., Irmen, F., Madsen, K.H., Haagensen, B.N., Schulze, S., Bisgaard, T., Siebner, H.R., Getting to grips with endoscopy - Learning endoscopic surgical skills induces bi-hemispheric plasticity of the grasping network, Neurolmage (2019), doi: https:// doi.org/10.1016/j.neuroimage.2018.12.030.

This is a PDF file of an unedited manuscript that has been accepted for publication. As a service to our customers we are providing this early version of the manuscript. The manuscript will undergo copyediting, typesetting, and review of the resulting proof before it is published in its final form. Please note that during the production process errors may be discovered which could affect the content, and all legal disclaimers that apply to the journal pertain. 


\section{Getting to grips with endoscopy - learning endoscopic}

2 surgical skills induces bi-hemispheric plasticity of the

\section{3 grasping network}

4

5

6 Anke Ninija Karabanov ${ }^{1 *}$, Friederike Irmen ${ }^{1,2}$, Kristoffer Hougaard Madsen ${ }^{1,3}$, Brian Numelin

7 Haagensen ${ }^{1}$, Svend Schulze ${ }^{4}$, Thue Bisgaard ${ }^{4}$, Hartwig Roman Siebner ${ }^{5,6}$ 8

$9{ }^{1}$ Danish Research Centre for Magnetic Resonance, Centre for Functional and Diagnostic 10 Imaging and Research, Copenhagen University Hospital Hvidovre, Denmark, Copenhagen

11 University Hospital Hvidovre, Hvidovre, Denmark

122 Berlin School of Mind and Brain, Humboldt-Universität zu Berlin, Germany

$13{ }^{3}$ Department of Applied Mathematics and Computer Science, Technical University of 14 Denmark, Denmark

$15{ }^{4}$ Gastrounit Surgical Division, Centre for Surgical Research (CSR), Copenhagen University 16 Hospital Hvidovre, Denmark

175 Department of Neurology, Copenhagen University Hospital Bispebjerg, Copenhagen, 18 Denmark

$19{ }^{6}$ Institute for Clinical Medicine, Faculty of Health and Medical Sciences, University of 20 Copenhagen, Copenhagen, Denmark

22 Classification: Biological Sciences

23

24

25

26

27

28 
1 Abstract

2 Endoscopic surgery requires skilled bimanual use of complex instruments that extend the

3 peri-personal workspace. To delineate brain structures involved in learning such surgical

4 skills, 48 medical students without surgical experience were randomly assigned to five

5 training sessions on a virtual-reality endoscopy simulator or to a non-training group. Brain

6 activity was probed with functional MRI while participants performed endoscopic tasks.

7 Repeated task performance in the scanner was sufficient to enhance task-related activity in

8 left ventral premotor cortex (PMv) and the anterior Intraparietal Sulcus (aIPS). Simulator

9 training induced additional increases in task-related activation in right PMv and aIPS and

10 reduced effective connectivity from left to right PMv. Skill improvement after training scaled

11 with stronger task-related activation of the lateral left primary motor hand area (M1-HAND).

12 The results suggest that a bilateral fronto-parietal grasping network and left M1-HAND are

13 engaged in bimanual learning of tool-based manipulations in an extended peri-personal space.

15 Keywords: Bimanual Skill Learning, Fronto-Parietal Grasping Network, Endoscopic Surgery 
1 Sensorimotor learning is fundamental to adapting our behavior to a changing environment

2 since it allows updating our interpretation of sensory information and to adjust our motor response (Makino et al., 2016). Learning a new motor skill, like manipulating a novel tool, is a special form of sensorimotor learning. It is behaviorally defined by the repetition-dependent increase in movement speed and accuracy for a given task (Diedrichsen and Kornysheva, 2015) and follows a distinct temporal pattern of fast initial improvements (early learning) and slower incremental gains (late learning) (Dayan and Cohen, 2011).

Neuroimaging has provided valuable insights into neural changes associated with sensorimotor skill learning (Hardwick et al., 2013) and most studies indicate that both cortical and subcortical motor areas (Primary Motor and Sensory Cortex (M1/S1), Premotor Cortex (PMC), Supplementary Motor Cortex (SMA), Cerebellum (CB), and Basal Ganglia (BG)) modulate their activity across training stages, often showing an initial increase in activity followed by a 'pruning' of activity during later learning (Dayan and Cohen, 2011; Doyon and Benali, 2005; Hardwick et al., 2013; Makino et al., 2016; Penhune and Steele, 2012; Steele and Penhune, 2010; Wymbs et al., 2012). Most studies that have traced skill learningdependent plasticity have relied on simple, unimanual sequences instead of complex toolbased skills (Floyer-Lea and Matthews, 2004; Karabanov et al., 2009; Karni et al., 1995; Lehericy et al., 2005; Penhune and Doyon, 2002; Puttemans et al., 2005; Steele and Penhune, 2010; Wymbs et al., 2012). Simple tasks lend themselves to the MRI environment but do not allow investigating finer grained training improvements on the continuum from novice to expert since behavioral improvements in these simple tasks plateau quickly. When investigating more complex sensorimotor skills involving elaborate bimanual manipulation of tools, functional brain mapping studies have often compared individuals with different experience levels (Doyon and Benali, 2005; Pinho et al., 2014). Tool manipulation activates a specific subset of cortical motor regions, also known as the ventrolateral grasping-network, consisting of the ventral Premotor Cortex (PMv), anterior Intraparietal Sulcus (aIPS), and primary motor hand area (M1-HAND) (Binkofski et al., 1999; Binkofski et al., 1998; Davare et al., 2011). While there is some evidence for a dominance of the left-hemispheric grasping network (Butler et al., 2004; Johnson-Frey et al., 2005; Stone and Gonzalez, 2014, 2015; Tsuda et al., 2009), inter-hemispheric interactions, especially during the acquisition of bimanual interactive skill with complex tools, are not well understood. 
1 Learning to use endoscopic instruments for minimally invasive surgery is a bimanual skill uniquely suited to study novice-to-expert transitions: Endoscopy requires the surgeons to grasp and manipulate delicate tissue structures by coordinated use of two elongated instruments, one operated by each hand. Hence endoscopic tools extend the peri-personal workspace and have to be used in a spatially restricted intra-operative setting. Appropriate use of these instruments requires finely tuned, visually and haptically guided bimanual movements in a technologically defined workspace (Louridas et al., 2016). Uncovering the neural correlates of surgical endoscopic skill improvement may aid the improvement of current training curricula. Evidence from earlier motor learning studies suggests that M1HAND may be especially relevant. In healthy human volunteers, early learning-related activity in contralateral M1-HAND predicted improved skill consolidation in a unilateral temporal sequence-learning task (Steele and Penhune, 2010) and has also been shown to increase during early learning of bimanual coordination tasks (Debaere et al., 2004; Puttemans et al., 2005). In monkeys, the lateral area in M1-HAND was identified to contain neuronal-subpopulation that are specialized for bimanual hand movements similar to those found in secondary motor areas (Aizawa et al., 1990).

To delineate the neural underpinnings of surgical endoscopic skills, several studies have attempted to map task-related brain activity during surgical laparoscopy in cross-sectional designs comparing different levels of expertise (Andreu-Perez et al., 2016; Duty et al., 2012; Leff et al., 2008; Ohuchida et al., 2009; Zhu et al., 2011). They found patterns of cortical activation consistent with the known pattern of early training activity expansion and late training activity pruning. Many functional mapping studies used near infrared spectroscopy (NIRS) focusing on prefrontal cortex, probably reflecting attentional control rather than aspects of sensorimotor control (Andreu-Perez et al., 2016; Leff et al., 2008; Ohuchida et al., 2009; Shewokis et al., 2017). The limited brain coverage and spatial resolution of NIRS precluded the investigation of especially relevant sensorimotor networks. We hypothesize that a ventral frontoparietal network, activated by both grasping movements (Binkofski et al., 1999; Binkofski et al., 1998) and tool use (Andres et al., 2017; Jacobs et al., 2010), and consisting out of the ventral premotor cortex (PMv) and the aIPS (Andres et al., 2017; Binkofski and Buxbaum, 2013), may play a critical role in acquiring endoscopic skills.

To trace brain correlates of endoscopic skill acquisition in the sensorimotor system, a MR- 
1 2014; Bahrami et al., 2011). In this study, we use an adapted version of that setup to trace

2 functional plasticity within tool-use related brain networks in a group of task-naïve medical

3 students across a 5-day training regime of basic endoscopic surgery skills on a virtual-reality

4 (VR) simulator. The aim of our study as twofold: First, we wanted to investigate functional plasticity within tool-use relevant ventral frontoparietal network during acquisition of a complex bimanual skill like surgical endoscopy. Second, we wanted to identify neural markers of training success within these networks.

\section{Methods}

\section{Participants}

Fifty-seven endoscopy inexperienced Danish medical students were recruited for the study and randomly assigned into training group (5 days of simulator training and 3 fMRI exams) or control group ( 3 fMRI exams only). Five participants were excluded due to incomplete data sets, one was excluded due to a technical failure leading to behavioral data not being recorded during the fMRI task, and three participants were excluded due to mistakes in fMRI image acquisition. Hence, the final sample for the fMRI analysis included 24 participants in the Training Group (mean age $24.0 \pm 1.8 ; 10$ females) and 24 participants in the Control Group (mean age $22.6 \pm 2.21 ; 14$ female). Participants were recruited by ads in a local student magazine and on university campus. All participants' handedness was assessed by using a handedness questionnaire adapted from the short Edinburgh Handedness Inventory (Veale, 2014) (85-90\% right-handers), they were currently enrolled in a Danish Medical Program, had no previous experience with performing, assisting or training endoscopic procedures, had no personal history of brain injury or illness and were not taking any neuroactive medication. All participants received detailed information and instructions about the experiment, signed informed consent and were reimbursed for their participation. The study was approved by the Regional Committee on Health Research Ethics of the Capitol Region in Denmark in accordance with the declaration of Helsinki.

\section{Experimental procedure}

Members of the Training Group trained once daily over five consecutive days on a standard, commercially available laparoscopy simulator outside the MR-scanner, completing a series of increasingly complex endoscopic excises for 40-50 min. Endoscopy-related brain activity was probed before, during and at the end of the 5-day training period by activating the taskrelevant networks in the MR-scanner via two simple exercises on an MR-compatible 
1 endoscopic box trainer (Figure 1A). The in-scanner box performance was intended to activate

2 the laparoscopy-related networks without providing the same complex learning experience as 3 the VR-simulator. A demographically similar control group completed the MR-laparoscopy sessions without further training (demographics in Table 1). Between-group comparison of brain activity during the in-scanner fMRI sessions enabled us to isolate training-induced changes in brain activity in the simulator-trained group from changes caused by repeated task performance during in-scanner fMRI-sessions. At the beginning of the study all participants also completed a set of questionnaires concerning handedness, experience with a range of manual motor actions (sports, instrument playing, video gaming, hand-crafting) and future medical specialization goals to ensure that the groups were evenly distributed on these parameters (See Table 1). A visualization of the entire experimental design can be seen in Figure 1.

\section{VR-simulator training}

Each VR-training session comprised a fixed training program based of 9 different exercises taken from the simulators basic tasks repertoire: We included right hand transfer, left hand transfer, bimanual transfer, grasping, lifting and grasping, cutting, fine dissection, precision and speed and suturing tasks in our training sequence as described in details elsewhere (see (Palter et al., 2012) for full list of LapSim tasks). Trained participants had to complete all exercises in the listed order and tasks were increasing in difficulty. Each of the tasks recorded a wide range of movement parameters monitoring performance speed, smoothness and accuracy (see (Woodrum et al., 2006) for more details on task metrics). All tasks, except for suturing were self-paced and ended upon task completion. Due to the difficulty of the final suturing task we set a performance limit of 3 minutes to complete the required two knots. During the first training session all training group participants received a thorough introduction to the training program and were instructed on how to complete all 9 exercises with a short demonstration and a trial run for each task under the supervision of an investigator. During all the recorded training sessions the participants were navigating through the training program themselves with an investigator in the room in case they had questions or technical problems. The investigator did however not provide feedback on their individual task performance. Performance during VR- training was quantified by generating an average performance score averaged over all tasks and task metrics. To be able to average each participant's performance across different task metrics, the single performance indicators 
1 subtracting the smallest value of the particular performance indicator in the whole group from each raw value and then dividing it by the metric's range. Performance metrics with very low variability (less than a total of 5 non-zero values across days and individuals were excluded

4 from the average rating. This mainly concerned a specific category of errors, measuring how often the instrument was outside of the camera view, which rarely happened. Removal of these measures was done because normalization would have artificially increased the weighting of these rare errors and these 'artificially inflated' normalized values would have contributed to the total score with the same weighting as variables with broad variance.

\section{In-scanner training}

During the fMRI protocol, subjects of both groups completed 4 task intervals of approximately 8 minutes each. In a block-design, participants alternated between 40 -second blocks of three different tasks: a) Simple Pointing, b) bimanual Endoscopic Transfer or c) rest. During 'Simple Pointing' the participants were instructed to alternatingly point the left instrument to the right rod and the right instrument to the left rod at a relatively steady pace that felt comfortable to them. During 'Endoscopic Transfer' participants were instructed to pick up a ring (with a ring diameter of $1 \mathrm{~cm}$ diameter) placed on one of the rods with one endoscopic instrument, transfer it to the other contralateral instrument/hand and place it on the second rod, once the ring was placed on the second rod it had to be moved back. One pickuptransfer-return movement sequence was defined as one cycle. Participants were instructed to complete as many correct cycles as possible within one 40-second loop. While movement instructions were different between tasks (comfortable pace for pointing vs. as many correct cycles as possible), the difference in task complexity level meant that the overall speed of movement was comparable. A transfer cycles consist of 4 separate movement trajectories (pick up (hand 1) - move to middle (hand 1) - move towards rod (hand2) - put down (hand 2)) while pointing consisted of a single movement trajectory (move towards rod). This means that very similar numbers of movement trajectories were executed in both conditions (pointing: $31.8 \pm 2.9$ (mean \pm std) vs. transfer: $8.17 \pm 5.31$ cycles, the cycle number multiplied by four resulted in 32.7 individual movement trajectories per transfer block. To further control for a possible confound of movement speed both intra- and inter individually we included the number of movements as a regressor in both our first and second level analysis (see Analysis of task-related fMRI data for details). During "rest", participants were instructed to keep the endoscopic instruments steady in their hands without any movement 
1 while keeping their eyes on the screen. The start of each block was indicated by written

2 instructions in the middle of the screen informing the participant about the next upcoming condition ('Pointing', 'Transfer', 'Rest'). In each session, the conditions were repeated 4 times. The order of task presentation was pseudo-randomized between sessions but stayed the same within a session. On Day 1 all participants were instructed both outside and inside of the scanner and instructed in how to perform all tasks with minimal arm movements. For the other scans participants received a short task and movement reminder before scan onset. Placement and fixation procedure was the same as for Scan 1.

\section{Apparatus}

11 VR-training: The training outside the scanner was done on a Surgical Simulator (LapSim 12 Haptic System, Surgical Science, Gothenburg, Sweden; http://www.surgical-science.com). 13 During training sessions participants' were standing upright in front of the stimulator and were manipulating a laparoscopic grasper comparable to the MR-compatible laparoscopic grasper in size, shape and function. Their actions were displayed in a 'virtual-reality' setting on the screen in front of them; they also received haptic feedback by the simulator when their instrument made contact with objects inside the virtual workspace. The out-of-scanner setup can be seen in figure $1 \mathrm{~B}$.

In-Scanner training: In-scanner training was conducted on a custom-made MR-compatible endoscopic box trainer (Bahrami et al., 2011). The participants were placed in supine position on the scanner bed with the box trainer placed on their lap at hip-height. A surgical (laparoscopic) instrument (40 cm long) was controlled by each hand. Participants were instructed to move the instruments with minor wrist and elbow movements exclusively in order minimize in-scanner movement. Supportive padding was placed under the participants' upper arms and elbows. Additionally, bandaging was placed around the upper arms and further restrained movement above the elbow. The workspace inside the box trainer contained a black platform $(7 \mathrm{~cm} \times 7 \mathrm{~cm})$ with two white rods $(4 \mathrm{~cm}$ apart $)$ with a red ring on each rod ( $2 \mathrm{~cm}$ in diameter). The platform and the rings were placed in the middle of the box, well reachable for the surgical instruments. The box workspace and all instrument movements were projected to a screen at the back of the scanner via a camera and a projector. The screen was visible to the participants via a mirror placed on top of the head coil. A visualization of the in-scanner setup is shown in figure 1A. Performance was also monitored on a screen in 
2 Differences between in-scanner and VR-training: Both tasks where designed to train tool-

3 based grasping and tool-based object manipulation skills required in endoscopy and activate

4 the brain networks associated with these skills. However, tasks were not identical in terms of body position (supine vs. upright), movement-range (restrained vs. free), task- complexity (select basic movements vs. full training program) and visual environment (fixed vs. changing dependent on task). The modification of VR-training during scanning was necessary to allow for the acquisition of BOLD data but limits measurable performance transfer between inscanner and VR-training session. While we expected both tasks to activate the same toolbased grasping network, we did not expect to measure strong skill transfer from the VRtraining to the scanner-sessions. In fact, were hoping for comparable in-scanner performance in the VR-training group and the control group as comparable behavioral performance would allow to interpret possible training-depended group differences in the BOLD data without considering the possible confound of group-specific differences in movement speed or accuracy.

VR-behavioural data: For the training group a wide range of performance variable measuring speed, movement accuracy and movement smoothness were recorded for the left and the right hand for each task during VR-training. To compute an overall mean performance score for each individual training session, the individual score for each performance variables was normalized by subtracting the minimum value of that variable in the task category (across days and subjects), the value was then divided by the standard derivation of the given variable such that all values were between 0 and 1 . Following within-variable normalization all measures were summed and divided by the total number of measures. This normalization allowed creating a combined 'session score' including information about movement smoothness, accuracy and speed for each task. Group data was checked for normality and after that a repeated measure ANOVA with the factor Time (Training Day 1 - 6) was calculated.

33 To compute an overall mean performance score for each training was normalized such that 34 values were between 0 and 1 . Following normalization all measures were summed and 
1 divided by the total number of measures. This normalization allowed creating a combined

2 'session score' including information about movement smoothness, accuracy and speed.

3 Group data was checked for normality and after that a repeated measure ANOVA with the

4 factor Time (Training Day 1 - 6) was calculated.

In-scanner behavioural data: For all participants the performance during the task-related fMRI session was video recorded. Performance during scanning sessions was visually scored for speed (number of correct ring transfers for transfer and number of left-right pointing for pointing) and accuracy (number of drops - accuracy measure for transfer only), leaving each participant with three performance measures per day (pointing_speed, transfer_speed, transfer_acc). Group data was checked for normality and after that mixed-measures ANOVAs with the factors Time (Scan1-3) and Group (Training vs. Control) were calculated for each dependent measure (resulting in three separate ANOVAs investigating pointing speed, transfer speed and transfer accuracy). These ANOVAs used the raw scores for in-scanner behaviour. To additionally investigate transfer between in-scanner and out-of scanner improvement in the VR-group speed and accuracy during in-scanner transfer were combined to the inverse efficiency score (IES) (Townsend and Ashby, 1978) and correlated with both the overall improvement of performance during VR training and with the transfer specific improvement during VR training using Pearson's product-moment correlations. For the IES, the improvement in transfer speed and accuracy ( $\Delta$ Last Session - First Session) was standardized (z-scored). The standardized transfer speed was divided by one (to reverse the scale) and subtracted by the standardized transfer accuracy.

\section{Image Acquisition}

26 Data acquisition was performed at the Danish Research Centre of Magnetic Resonance, Hvidovre Hospital, Copenhagen, Denmark on a 3T Achieva scanner (Philips). A scanning session consisted of a structural T1-weighted sequence (MPRAGE; FOV: 245mm; 245 slices, TR/TE: 5.9/2.7; resolution $0.85 \times 0.85 \times 0.85 \mathrm{~mm}^{3}$; flip angle: $8 \mathrm{deg}$.; TI: $747.6 \mathrm{~ms}$ ), one restingstate T2*-weighted fMRI sequence (EPI; FOV: 192mm; 32 slices: TR/TE: 2000/30; in-plane resolution: $3 \times 3 \mathrm{~mm}^{2}$, $3 \mathrm{~mm}$ slice thickness, $1 \mathrm{~mm}$ slice gap, flip angle: 90 deg.), four taskrelated T2*-weighted fMRI sessions (EPI; FOV: 192mm; 32 slices: TR/TE: 2000/30; in-plane resolution: $3 \times 3 \mathrm{~mm}^{2}$, $3 \mathrm{~mm}$ slices, $1 \mathrm{~mm}$ slice gap, flip angle: 90 deg.) and three diffusion- 

$2.3 \times 2.3 \times 2.3 \mathrm{~mm}^{3}$, flip angle: $8 \mathrm{deg} . \quad b=2000 \mathrm{~s} / \mathrm{mm}^{2}$ (62 directions), $b=1000 \mathrm{~s} / \mathrm{mm}^{2}(62$ directions), $b=300 \mathrm{~s} / \mathrm{mm}^{2}$ (6 directions). A bipolar gradient scheme was used to allow for model-based eddy current correction. Additional $b_{0}$ references for each sequence were acquired with both A-P and P-A phase encoding for correction of susceptibility artefacts (topup in FSL), up to 62 directions.

\section{Image data analysis}

Pre-processing

The fMRI data were analyzed using Statistical Parametric Mapping (SPM12; Wellcome Trust Centre for Neuroimaging, London, UK) implemented in MATLAB 8.3.0 (MathWorks).

First, both the MPRAGE and the EPI sequences were manually reoriented according to the location and orientation of the anterior/posterior commissures. After that, all EPI images were slice time corrected using the temporal center slice as reference and realigned to the mean EPI image (within every session) to correct for small head movements using a two-step procedure. The resulting images were normalized to a standard EPI template based on the Montreal Neurological Institute (MNI) reference brain using an affine 12-parameter transform and a discrete-cosine based nonlinear transformation. Images were then resampled to $2 \mathrm{~mm}^{3}$ voxels, and smoothed with an isotropic $6 \mathrm{~mm}$ full-width half-maximum Gaussian kernel to decrease residual inter-subject differences and to increase the signal-to-noise ratio.

Analysis of task-related fMRI data: The statistical analysis of task-related fMRI data was performed using the mass-univariate general linear model (GLM). The design matrix was generated with a synthetic hemodynamic response function and a set of 24 motion regressors modeled using an expansion of the estimated movement parameters from the rigid body realignment procedure (six parameters) and motion parameters from the previous volume, squared motion parameters, and squared motion parameters from the previous volume (Friston et al., 1996). Additionally, a high-pass filter for removal of low-frequency trends originating from scanner drift and three performance regressors coding for movement speed (for pointing and transfer separately) and ring drops (for endoscopic Transfer) were included as parametric modulations in order to model low-level effects of movement speed or errors. The two regressors of interest modeled in each session were the Simple Pointing task (functioning as a high-level motor control) and the complex bimanual endoscopic Transfer. The rest condition was not modeled directly but included as an indirect baseline. The effect of Transfer (Transfer $>0$ ), Pointing (Pointing $>0$ ) and the effect of Transfer against Pointing 
1 (Transfer > Pointing) were analyzed by computing the respective contrasts on the first level

2 for each participant and scan session independently. Since the complex bimanual transfer is reminiscent of the exercises from the VR-training, the contrast Transfer > Pointing for each scanning session and subject was used in a summary statistic random effects factorial design with the factors Group (assumed independence, unequal variance) and Time (assumed dependence, unequal variance) that allowed us to test for main effects of Time and Group, non-spherical errors were handled by pre-whitening through the restricted maximum likelihood procedure implemented in SPM12. To remove any possible movement-induced confounds across subjects, time or group, the individual number of pointing movements and transfer cycles during each scanning session were included in the design as two separate regressors of no interest when comparing experimental groups directly. To localize regions in which Time led to a BOLD signal modulation specifically for the Training Group, we also used contrasts assuming a linear signal change in the training group with a stationary signal in the control group. We pre-defined the PMv and AIP bilaterally as regions of interest (RoIs), because these regions have been previously identified as core regions of a ventral frontoparietal grasping loop (Binkofski et al., 1999). The RoIs were defined as 35-mm spheres centred on the PMv and AIP coordinates as reported in (Binkofski et al., 1999). For these ROIs, we applied small volume correction using the family-wise error correction method as implemented in SPM12 (threshold set at $\mathrm{p}<0.05$ ).

To check if the BOLD signal could either predict or explain the results from the VRbehavioural data for the training group (see Results), we then conducted correlational analysis using either final performance on the box trainer, the individual improvement in out-ofscanner training (Training day 5 - day 1 ) or the final score during out-of-scanner performance as a co-regressor and correlating those with either with individual BOLD during the TrainingPointing contrast at baseline or with individual BOLD change (Scan 3 - Scan 1) in the same contrast. For the correlational analysis we applied small volume correction before family-wise error correction based on our a-priori hypothesis on the importance of M1 (Aizawa et al., 1990; Steele and Penhune, 2010). Small volume correction was done using a 35-mm sphere around the M1 coordinates [-42 -25 65]) implicated in retention by earlier work (Steele and Penhune, 2010). 
1 multivariate analysis. DCM models hidden dynamics underlying changes in fMRI signal

2 based on differential equations, comprising an A-matrix (baseline coupling), a B-matrix

3 (contextual modulation of the connections; Time), and a C-matrix (direct inputs to the system;

4 here Tasks; (Friston et al., 2003)). Based on the local activation peaks from the fMRI contrast 'Linear Training Increase' we constructed a DCM including the bilateral PMv and aIPS activation peaks implicated by the GLM analysis (PMv MNI: 54, 8, 29; -54, 5, 35; aIPS MNI: $63-1937,-54,-25,35$; all $\mathrm{p}$ uncorrected < 0.001). The first eigenvariate representing the fMRI time series were extracted from all voxels within a $6 \mathrm{~mm}$ radius of these coordinates from each subject. We included a direct reciprocal connection inter-hemispherically between left and right PMv and aIPS (Boussaoud et al., 2005; Manzoni et al., 1989) as well as interhemispheric connections between PMv and aIPS on each side (Dancause et al., 2006; Gharbawie et al., 2011; Ghosh and Gattera, 1995). Input (C-matrix) was set to aIPS, because it is assumed that for conscious intentional movements, aIPS activity precedes activation of the PMv (Desmurget and Sirigu, 2009). We created and compared several alternative models (Figure 4). Their critical difference lay first in whether training, task, or both modulated fronto-parietal connectivity and second in whether intrahemispheric connections, interhemispheric connections, or both were modulated. We also included a null model, which postulated that there was no modulation of connectivity. This resulted in 8 different models for each participant. To model Training as a modulatory input to the DCM, fMRI data of all three days were concatenated in a new GLM and all models were then fitted to the fMRI data for both the training and control group. The model included the same conditions as the first level GLM described above. The eigenvariate for the conditions pointing and transfer was extracted from the Volumes of Interest in the coordinates described above and adjusted for the overall main effect. Bayesian model selection (BMS) was used to detect the model that best explained the data taking into account model complexity (Penny et al., 2004) where differences in free energy (F) indicate evidence for a given model (Friston et al., 2007) . After having identified the most likely model for each group (training/no-training), the connectivity parameters (B-matrix) of the winning model were extracted to test whether modulation of bilateral parieto-frontal connectivity showed a significant Time modulation. 
Results

2

\section{Behavioral performance}

4 Five-Day endoscopic training program on simulation trainer: The averaged, daily performance score for each individual in the training group showed that the group steadily increased their performance during the training sessions (Figure 2; $F(4)=53.8 ; p<0.0001$; repeated-measures ANOVA; repeated factor: performance index, Training Session 1-5). Posthoc tests (Fisher's least significant difference (LSD)) confirmed a significant day-to-day increase throughout the first 4 sessions (D1-D2, D1-D3, D1-D4, D1-D5 all p< 0.0001; D2 D3, $\mathrm{p}=0.027$; D2-D4, D2-D5 both $\mathrm{p}<0.0001$; D3-D4, $\mathrm{p}=0.023$, D3-D5, $\mathrm{p}=0.001$; D4-D5, $\mathrm{p}=0.2)$

Endoscopic Performance during fMRI: For the pointing task, performance was indexed by the mean number of left-right pointing movements (speed measure) per task block. Task performance for transfer was approximated by the mean number of transfer cycles (speed measure) and mean number of ring drops (accuracy measure) per task block. The ANOVAs for both, pointing speed and transfer speed showed that both groups improved their performance speed during pointing and transfer $(\mathrm{F}(2,47)=34.8 ; \mathrm{p}<0.0001$ and $\mathrm{F}(2,47)=$ 43.8; $\mathrm{p}<0.0001$ respectively; mixed-effects ANOVAs). For transfer the increase in speed was not accompanied by an drop in accuracy as the ANOVA testing accuracy during transfer did not show a main effect of time $(F(2,47)=2.6 ; p=0.08)$. None of the three ANOVAs did show a significant effect for group or a Group x Time interaction (all values $\mathrm{p}>0.2$; Figure 3 ) indicating that both groups performed comparable throughout scanning sessions

Relationship between simulator training and inside scanner performance: While a Pearson's product-moment correlation between the total Improvement Score on the VR-Simulator $(\Delta$ Last Session - First Session) and improvement during in scanner performance, estimated by the inverse efficiency score (IES) combining standardized speed and accuracy during inscanner transfer (Townsend and Ashby, 1978), was non-significant ( $\mathrm{p}>0.2$ ), a planned correlation between the VR-Improvement Score for Transfer alone and the IES did show a moderate correlation ( $r=0.39 ; \mathrm{p}=0.05$ ) (Supplementary Figure 1a). We also asked a sub-set $(\mathrm{N}=13)$ of control participant to take part in a session of the VR-simulator training after they had completed all three scanning sessions. Comparing the performance during the single 
1 showed that performing the simple laparoscopy exercises during scanning did not result in

2 improved VR-simulator performance. Simulator performance in the sub-sample of the control

3 group was significantly worse than the performance of the training group both when

4 compared to the final performance score obtained in the $5^{\text {th }}$ training session $(\mathrm{t}(35)=-6.13 ; \mathrm{p}<$

5 0.0001) and when compared to the first training session $(\mathrm{t}(35)=-2.61 ; \mathrm{p}=0.01)$

6 (Supplementary Figure 1b).

\section{Laparoscopy-related brain activity}

9 To reveal the whole-brain network activated by typical endoscopic movement sequences at 10 baseline, voxel-wise activation patterns were compared between the bimanual 'Endoscopic 11 Transfer' task and the Simple Pointing' task within the initial fMRI exam (Baseline Scan). 12 Both groups were included in this analysis, because all participants (training/control group) 13 were equally naïve to endoscopic procedures at baseline. Endoscopic transfer manipulations, 14 relative to simple pointing, activated bilateral clusters of parietal and occipital regions related to visuo-motor coordination including a cluster including the occipital portion of the fusiform gyrus and the inferior occipital gyrus spanning into the middle temporal gyrus and motion area in V5 as well as a cluster in the superior parietal lobule (Figure 4A; Table 2). Somatosensory areas subserving haptic processing during tool manipulation (primary sensory cortex (S1)) were also activated. The activation map also revealed a large bilateral cerebellar cluster and bilateral clusters in frontal motor areas, including primary motor cortex (M1) and dorsal premotor cortex $(\mathrm{PMd})$. The inverse analysis revealed that bilateral regions of the angular gyrus, medial frontal and prefrontal cortex, hippocampus and anterior cingulate cortex were more activated during simple unimanual pointing compared to bimanual transfer (See Supplementary Table 1). These areas are usually associated with memory, higher cognitive functions and pace keeping and may be explained by the fact that participants were asked to keep a steady pace during pointing. At baseline there were no significant differences in the activation maps of the two groups (F-test, highest activation peak at 0.001 uncorrected: 9 voxels at $\mathrm{x}=-27 ; \mathrm{y}=14, \mathrm{z}=65 ; \mathrm{FEW}$-corrected $\mathrm{p}$-value $=0.94)$

\section{Time-dependent change in task-related activity independent of simulator training}

31 Both groups performed the in-scanner endoscopic task blocks during three fMRI examinations. To delineate general changes in task-related activity during repeated withinscanner endoscopic performance, we contrasted voxel-wise activation maps (Endoscopic 
1 task-related brain activity changed over time. Voxel-wise activation ( $\beta$ ) patterns were

2 compared between Scan 1 (baseline) and Scan 2 (day 2) and between Scan 1 (baseline) and

3 Scan 3 (day 6) using family-wise error correction (voxel-wise; $\mathrm{p}<0.05$ ). During early training

4 (Scan 2 - Scan 1), we observed 4 distinct clusters showing a relative increase in activation

5 with bimanual transfer. These clusters were located in the left anterior putamen $[\mathrm{t}=5.11 ; \mathrm{x}=$

$6-15 ; \mathrm{y}=5, \mathrm{z}=-4]$, and bilaterally at the border between ventral premotor cortex (PMv) and

7 the inferior frontal gyrus (IFG) $[\mathrm{t}=4.82 ; \mathrm{x}=-54 ; \mathrm{y}=5, \mathrm{z}=20$ and $\mathrm{t}=4.29 ; \mathrm{x}=54 ; \mathrm{y}=5 ; \mathrm{z}=$

8 20] (Figure 4B) and in the right dorsal premotor cortex $[\mathrm{t}=4.22 ; \mathrm{x}=30 ; \mathrm{y}=-7 ; \mathrm{z}=-47]$. The

9 comparison between baseline and the third fMRI session showed that the full left grasping 10 pathway, cluster in left PMv $[\mathrm{t}=5.17 ; \mathrm{x}=-48 ; \mathrm{y}=1, \mathrm{z}=26]$ and a cluster in the left aIPS ([t $11=4.66 ; \mathrm{x}=-57 ; \mathrm{y}=-25 \mathrm{z}=29$ ], displayed significant increase over time. No brain region 12 showed decreasing activity with bilateral transfer over the three scanning sessions. In 13 summary, the results show that short periods of skill exercise during fMRI was sufficient to 14 engage the left-hemispheric ventral fronto-parietal grasping network along with the anterior 15 putamen. (Figure 4b).

\section{Time-dependent change in task-related activity specific to simulator training}

To test how five-day VR-simulator training, in addition to task performance during the three fMRI sessions, altered task-related brain activity we compared voxel-wise activation maps using a contrast defining a linear increase across days in the training group and steady activation in the control group. Two right-hemispheric areas in ventral frontoparietal cortex showed an increase in task-related activation in the training group relative to the control group (Fig.5). The first cluster was located at the border between right PMv and IFG (BA44) $[\mathrm{t}=$ 4.69; $\mathrm{x}=54 ; \mathrm{y}=8, \mathrm{z}=29]$. The second cluster was located at the border between ventral primary sensory cortex (S1) and aIPS $[\mathrm{t}=4.20 ; \mathrm{x}=63 ; \mathrm{y}=-19, \mathrm{z}=37]$. Both clusters were part of the ventral frontoparietal grasping network (Binkofski et al., 1999). Interestingly, the location of the two right-hemispheric clusters showing a training-specific increase in activation precisely matched the locations of the two left-hemispheric clusters which increased their task-related activations in both groups due to repeated task performance in the MR scanner. Together, the task-related fMRI activation data show a bilateral involvement of the ventral PMv-aIPS network during the acquisition of surgical endoscopic skills with righthemispheric recruitment being specific during VR-based skill training (Figure 5).

\section{Training-related change in effective connectivity}


1 To model the network dynamics underlying the changes in fMRI signal over time, Dynamic

2 Causal Modeling (DCM) was performed. We specified and compared eight different models 3 (Figure 5A), which differed in terms of time-dependent modulation of functional 4 connectivity between the four included areas. First, BMS (Baysian Model Selection) (Penny et al., 2004) was used to compare models with different connectivity profiles and to find the model that best explained the observed data. Since the GLM analysis indicated a different modulatory effect of time between the groups, model selection was run separately for the training and control group. While both models agreed on choosing the same family of interhemispheric models, two different interhemispheric network models best explained the data for the control group and the training group. For the control group a model with connections between left and right aIPS best explained the data (Figure 6B, Model 3), while a model with connections between left and right PMv and between left and right aIPS best explained the data for the training group (Figure 6B, model 4). The posterior probability of the winning model was $>99.8 \%$ with a Bayes Factor above 10 for the training group and above 99.9\% with a Bayes Factor above 10 for the control group. Since the same model did not best explain effective connectivity patterns of the two groups, we based further analysis of the modulatory effect of training on the training group alone and the corresponding network parameters of model 4. A repeated-measures ANOVA of the connectivity values of coupling modulation by training revealed a significant decrease of coupling from the left to the right PMv (Figure 4C; F (2) = 3.2 p < 0.047; repeated factor: Scanning Session 1-3). Also, for the other three connections (right PMv $\rightarrow$ left PMv, right aIPS $\rightarrow$ left aIPS, left aIPS $\rightarrow$ right aIPS a nominal, but not statistically significant decrease in coupling over fMRI sessions was observed ( $p=0.12, p=0.21$ and $p=0.25$ respectively). The change in coupling values did not correlate with individual performance gains over the training period.

\section{Relationship between training-related performance gain and task-related activation}

Using multiple regression analysis, we tested which voxel-wise activation patterns were predictive of training success in the training group. Task-related activation maps at baseline did not scale with performance improvement or absolute final performance. In contrast, we identified one cluster where individual time-dependent changes in task-related activation from pre- to post-training changed in proportion with performance improvement during simulator training. In the lateral and anterior portion of left M1-HAND, a discrete cluster 
baseline scan 1 to scan 3 in proportion with the individual performance improvement during the simulator training $(\mathrm{r}=0.72, \mathrm{p}<0.001$; Cluster: $\mathrm{x}=-42 ; \mathrm{y}=-25, \mathrm{z}=65$ and $\mathrm{t}=4.97$,

3 Figure 7).

4

\section{Discussion}

Here we combined state-of-the VR-based simulator training in the laboratory with taskrelated fMRI in a simplified endoscopic set-up to study experience-dependent plasticity in the human grasping network. Endoscopic surgery requires finely tuned bimanual manipulation of surgical tools in a highly constrained workspace. A particular feature of endoscopic surgical training is that the tools are elongated, significantly expanding the peri-personal workspace. This functional extension of the upper limbs alters the body schema, defined as the pragmatic sensorimotor representation of the body's spatial properties. When naïve individuals became acquainted with the bimanual use of endoscopic surgical tools, the grasping network, most notably the PMv, underwent an experience-dependent shift. While simple task-repetition during scanning was sufficient to increase activity in the left (dominant) ventrolateral grasping pathway, including PMv and aIPS, a five-day course of simulator training prompted a bilateral increase in functional engagement of this pathway along with a down-regulation of inter-hemispheric coupling from left to right PMv. While the grasping network displayed consistent training-dependent activity changes, it was task-related activity in a lateral cluster of the left M1-HAND, which scaled with the individual improvement in endoscopic surgical skill.

\section{Five-day training versus repeated task exposure in the MRI scanner}

Our task design allowed us to differentiate between training-induced sensorimotor plasticity induced by a five-day VR-simulator training and alterations in sensorimotor activity induced by the repeated performance of simple endoscopic tasks in the fMRI sessions. On a neural level, both groups showed increasing task-related activity in the left PMv and aIPS, but only the training group also showed increasing task-related activity right hemispheric homologous areas implicating the bilateral ventrolateral grasping pathway (Binkofski and Buxbaum, 2013; Rizzolatti and Matelli, 2003). The ventrolateral grasping pathway is involved in the abstract representation of action goals and has been shown to decode goal-directed object manipulation irrespective of whether a hand or a tool was used as end-effector in both, monkeys and humans (Arbib et al., 2009; Gallivan et al., 2013; Garcea and Mahon, 2014; 
1 Martin et al., 2011; Ochiai et al., 2005). To the best of our knowledge, we are the first to show that learning novel hand-to-tool transformations is accompanied with bilaterally increasing activity in the ventrolateral grasping pathway over the course of training but many studies have reported activity of the left-hemispheric ventrolateral grasping pathway in a single scanning session. These studies have reported a left-dominant tool-use activation pattern independent of handedness and activated hand (Frey, 2008; Jacobs et al., 2010; Martin et al., 2011) though some research suggest that left-handers may activate a bilateral network during tool-based grasping (Martin et al., 2011). The current study suggests that already repeated exposure to a novel tool-dependent skill in the neuroimaging sessions is sufficient to engage this left-dominant network, indicating that some aspects of hand-to-tool transformation become quickly represented within the left-sided network (Brozzoli et al., 2011; di Pellegrino and Ladavas, 2015; Sengul et al., 2012). The effects related to repeated task-exposure revealed by the current study emphasize the importance of adequate non-training control groups when evaluating training interventions on task-related activity as a simple withinsubject comparisons would have not been able to differentiate between VR-related and taskrepetition related effects. The use of movement regressors in both individual GLMs and in the summary statistics has also allowed us to exclude that the observed effects were due to the amount of movement rather than training-dependent changes in BOLD signal.

Only the training group showed an additional engagement of the right-sided PMv and aIPS areas. Literature on predominantly right-sided activation of the ventrolateral grasping pathway is scarce, but there is evidence for predominantly right-sided activations during haptically guided grasping movements (Marangon et al., 2015). Speculatively, this may indicate that VR-training aided the participants to better integrate haptic feedback on positioning (e.g. whether instruments are touching each other, the rings, the environment ect.) into their action plans. Anecdotal self-report from the training group, where some participants reported that they where starting to rely more on proprioceptive feedback over the course of training supports this hypothesis.

Behaviorally, we were able to see that improvements during the transfer task in the VRenvironment were predictive of transfer improvements in the MR-environment. However, general improvements across the full VR-training battery were not predictive of the inscanner performance and VR-training did not result in a significantly better in-scanner 
1 of behavioral output in the scanner the observed, training-associated changes in the bilateral

2 grasping network indicate that the observed network effect of VR-training may reflect modulations in task 'framing' (e.g. changed weighting of sensory information or of dominant/non-dominant effector) or effort that are independent of direct behavioral output and similar intrinsic chances in cerebral activating patterns have also been found in other studies investigating visually guided bimanual training (Remy et al., 2008). Together this data suggests that skill improvements may be highly task specific and further studies should investigate inter-task transfer and the role of task framing more closely. Additionally it is possible that the constraints necessary to enable task performance in the scanner (supine positioning, movement restraints) may have affected task transfer and future studies, interested in transfer, may consider keeping such variables identical across within and out-of scanner settings.

While discovering time-dependent increases in task-related activations, we were not able to observe any brain regions where task-related activation decreased over time. A gradual pruning of activity, indicating more efficient sensorimotor processing and increased automation, is often observed when healthy individuals acquire a novel sensorimotor skill. Of note, performance in the endoscopic surgical task did not reach a performance plateau. Therefore, we attribute the absence of any decreases in task-related activity to the fact that participants did not reach skill automatization during the course of the study.

\section{Inter-hemispheric interactions within the grasping network}

23 Effective connectivity analysis was applied to further investigate effective coupling in the bihemispheric ventrolateral grasping network. DCM analysis was able to show that functional impact of the left PMv on to the right PMv significantly decreased over time in the training group. For the control group, the winning model did not include coupling between PMv nodes and hence modulation over time was not investigated in this group. Structurally, direct interhemispheric connections of the left and right PMv have been demonstrated both in monkeys (Dancause et al., 2006) and humans (Park and Hallett, 2015). Functionally, TMS investigations suggest that activating the contralateral PMv can weaken the influence the ipsilateral PMv has on M1-HAND (Park and Hallett, 2015). We hypothesize that, in the context of bilateral training, a reduced drive from the left to the right PMv 'released' the right $\mathrm{PMv}$ from the inhibitory influence exerted by the left PMv on its right hemispheric 


\section{Recruitment of left M1-HAND scales with performance improvement}

3 Several studies have implicated the importance of M1-HAND in unimanual skill

4 improvement and consolidation (Muellbacher et al., 2002; Steele and Penhune, 2010). Yet, 5 for bimanual skills, the predominant view has been that the supplementary motor area (SMA)

6 rather than M1-HAND is critical for optimal coordination of hands (Halsband and Lange, 7 2006; Jancke et al., 2000). This view has been challenged by several neurophysiological 8 studies showing the existence of large populations within M1-HAND that are specific to 9 bimanual movements (Aizawa et al., 1990; Donchin et al., 1998; Gribova et al., 2002). 10 Extending these findings, we identified a lateral cluster in the human M1-HAND where task11 related activity scaled with the individual improvement in the acquired surgical skill. The 12 anatomical location of this lateral cluster in the anterior bank of the left M1-HAND is in good 13 agreement with the reported location of bimanual sensitive M1 neurons in monkey and with 14 the location shown to predict unimanual training outcome in humans (Aizawa et al., 1990; 15 Steele and Penhune, 2010). It is possible, that bimanual control in M1 is stronger for arm than 16 for finger movements as several studies on trans-callosal projections between motor cortices have found that areas for arm representation are much stronger connected than areas of hand representation (Jenny, 1979; Muakkassa and Strick, 1979; Rouiller et al., 1994).

\section{Task-related activation of other brain areas}

21 A bilateral dorsal fronto-parietal network associated with visual guidance of actions including the SPL and PMd was activated in all participants throughout all fMRI sessions. These areas are activated by a range of sensory-guided motor tasks, and adjust force based on object features during grasping movements (Binkofski and Buxbaum, 2013; Brandi et al., 2014; Davare et al., 2011; Rizzolatti and Matelli, 2003; van Nuenen et al., 2012; van Polanen and Davare, 2015). As sensorimotor integration was a constant requirement throughout all training sessions, activity in these areas remained high throughout the entire study. Participants also showed robust cerebellar activity from the first endoscopic performance indicating the importance of error-based feedback during the task (Diedrichsen et al., 2010; Makino et al., 2016). We hypothesize that these areas show gradual attenuation in task-related activation with more prolonged training along with increasing automatization of endoscopic surgical skills. 
1 We observed transient task-exposure related activity increase in the left anterior putamen and

2 the right PMd. In both groups, activity in these regions transiently increased during the second exposure to in-scanner laparoscopy, but had returned to baseline levels at the time of the third fMRI session. The short-term modulation of the putamen and PMd may indicate that associative sensorimotor learning, primarily mediated via the cortico-striatal motor loop connecting premotor areas with the anterior putamen (Seger, 2006) already took place during the first training sessions. Many theories on the neuroplasticity sub-serving motor learning emphasize learning related activity in- and decreases (Dayan et al., 2013), yet we have not been able to find any learning-related activity decreases in the current study. We believe this can be explained by the relative complexity of the task: Performance in neither in-scanner not VR-training reached an improvement plateau, characteristic of the end of the early learning phase. In this sense the time course of the present study is different when compared to a row of studies investigating much simple, rhythmic, bimanual coordination tasks, where asymptotic behavior is typically reached within a single training session (Debaere et al., 2004; Puttemans et al., 2005). It is worth noting that these studies consistently reported the dorsal premotor cortex to display decreasing activity in bimanual training. Our own neuroimaging work has also consistently shown an engagement of the dorsal premotor cortex in visually guided unimanual hand movements, especially in a non-routine context (Hartwigsen et al., 2012; van Eimeren et al., 2006; van Nuenen et al., 2012; Ward et al., 2010). In the present study, the dorsal premotor cortex was part of the general task-related network at Day1 and also showed a transient increase in task-related activation from Day1-2. It is possible to assume that continuation of training would reveal a decrease in that area beyond the level recorded at baseline once the endoscopic skills becomes automatic.

\section{Implications for surgery training}

One of our experimental hypotheses was that skill improvement could be predicted by BOLD activity during the baseline scan but prediction of behavioral improvement, was not possible when using only the initial scans. While our work suggests that fMRI at pre-training baseline cannot be used to predict endoscopic training success, interesting conclusions may be drawn from uncovering brain activity over the course of learning. Our work suggests that already short periods of endoscopic task exposure are enough to extend the peri-personal space by incorporating the endoscopic instruments and engage the left-hemispheric grasping network. However, additional VR-training was required to produce a balanced bilateral increase of the grasping network during the performance of basic movement components. The balanced 
1 activation may aid bimanual control of the dominant and non-dominant hand during real-life

2 endoscopic task performance.

3

4

5

6

\section{Acknowledgements}

We would like to thank Mei-Yun Zheng and Anuza Mayendraraj who helped with VR surgery training and with evaluation of in-scanner task performance. This work was supported by the Lundbeck Foundation (Grant of Excellence: Mapping, Modulation \& Modeling the Control of Actions; Grant nr. 59-A5399) and Toyota Fonden (Grant nr. KJ/BG 8686 H). Hartwig R. Siebner holds a clinical professorship in precision medicine at Copenhagen University sponsored by the Lundbeck Foundation.

\section{Competing Interests}

Hartwig R. Siebner has received honoraria as speaker and consultant from Sanofi Genzyme, Denmark and as senior editor (NeuroImage) from Elsevier Publishers, Amsterdam, The Netherlands. He has received royalities as book editor from Springer Publishers, Stuttgart, Germany and has received research funding from Biogen-idec, Denmark. The other authors report no conflict of interests,

\section{References}

Aizawa, H., Mushiake, H., Inase, M., Tanji, J., 1990. An output zone of the monkey primary motor cortex specialized for bilateral hand movement. Exp Brain Res 82, 219-221. 
Andres, M., Pelgrims, B., Olivier, E., Vannuscorps, G., 2017. The left supramarginal gyrus contributes to finger positioning for object use: a neuronavigated transcranial magnetic stimulation study. Eur J Neurosci 46, 2835-2843.

Andreu-Perez, J., Leff, D.R., Shetty, K., Darzi, A., Yang, G.Z., 2016. Disparity in Frontal Lobe Connectivity on a Complex Bimanual Motor Task Aids in Classification of Operator Skill Level. Brain Connect 6, 375-388.

Arbib, M.A., Bonaiuto, J.B., Jacobs, S., Frey, S.H., 2009. Tool use and the distalization of the end-effector. Psychol Res 73, 441-462.

Bahrami, P., Graham, S.J., Grantcharov, T.P., Cusimano, M.D., Rotstein, O.D., Mansur, A., Schweizer, T.A., 2014. Neuroanatomical correlates of laparoscopic surgery training. Surg Endosc 28, 2189-2198.

Bahrami, P., Schweizer, T.A., Tam, F., Grantcharov, T.P., Cusimano, M.D., Graham, S.J., 2011. Functional MRI-compatible laparoscopic surgery training simulator. Magn Reson Med 65, 873-881.

Binkofski, F., Buccino, G., Stephan, K.M., Rizzolatti, G., Seitz, R.J., Freund, H.J., 1999. A parieto-premotor network for object manipulation: evidence from neuroimaging. Exp Brain Res 128, 210-213.

Binkofski, F., Buxbaum, L.J., 2013. Two action systems in the human brain. Brain Lang $127,222-229$.

Binkofski, F., Dohle, C., Posse, S., Stephan, K.M., Hefter, H., Seitz, R.J., Freund, H.J., 1998. Human anterior intraparietal area subserves prehension: a combined lesion and functional MRI activation study. Neurology 50, 1253-1259.

Boussaoud, D., Tanne-Gariepy, J., Wannier, T., Rouiller, E.M., 2005. Callosal connections of dorsal versus ventral premotor areas in the macaque monkey: a multiple retrograde tracing study. BMC Neurosci 6, 67.

Brandi, M.L., Wohlschlager, A., Sorg, C., Hermsdorfer, J., 2014. The neural correlates of planning and executing actual tool use. J Neurosci 34, 13183-13194.

Brozzoli, C., Gentile, G., Petkova, V.I., Ehrsson, H.H., 2011. FMRI adaptation reveals a cortical mechanism for the coding of space near the hand. J Neurosci 31, 9023-9031.

Butler, A.J., Fink, G.R., Dohle, C., Wunderlich, G., Tellmann, L., Seitz, R.J., Zilles, K., Freund, H.J., 2004. Neural mechanisms underlying reaching for remembered targets cued kinesthetically or visually in left or right hemispace. Hum Brain Mapp 21, 165-177.

Dancause, N., Barbay, S., Frost, S.B., Plautz, E.J., Stowe, A.M., Friel, K.M., Nudo, R.J., 2006. Ipsilateral connections of the ventral premotor cortex in a new world primate. J Comp Neurol 495, 374-390.

Davare, M., Kraskov, A., Rothwell, J.C., Lemon, R.N., 2011. Interactions between areas of the cortical grasping network. Curr Opin Neurobiol 21, 565-570. Dayan, E., Censor, N., Buch, E.R., Sandrini, M., Cohen, L.G., 2013. Noninvasive brain stimulation: from physiology to network dynamics and back. Nat Neurosci 16, 838-844. Dayan, E., Cohen, L.G., 2011. Neuroplasticity subserving motor skill learning. Neuron 72, 443-454. brain activation during the acquisition of a new bimanual coodination task. Neuropsychologia 42, 855-867.

Desmurget, M., Sirigu, A., 2009. A parietal-premotor network for movement intention and motor awareness. Trends Cogn Sci 13, 411-419. di Pellegrino, G., Ladavas, E., 2015. Peripersonal space in the brain. Neuropsychologia 66, 126-133.

49 Diedrichsen, J., Kornysheva, K., 2015. Motor skill learning between selection and execution. Trends Cogn Sci 19, 227-233. 
Diedrichsen, J., White, O., Newman, D., Lally, N., 2010. Use-dependent and error-based learning of motor behaviors. J Neurosci 30, 5159-5166.

3 Donchin, O., Gribova, A., Steinberg, O., Bergman, H., Vaadia, E., 1998. Primary motor 4 cortex is involved in bimanual coordination. Nature 395, 274-278.

Doyon, J., Benali, H., 2005. Reorganization and plasticity in the adult brain during learning of motor skills. Curr Opin Neurobiol 15, 161-167.

Duty, B., Andonian, S., Ma, Y., Peng, S., Shapiro, E., Dhawan, V., Richstone, L., Eidelberg, D., Kavoussi, L.R., 2012. Correlation of laparoscopic experience with differential functional brain activation: a positron emission tomography study with oxygen 15-labeled water. Arch Surg 147, 627-632. with increased movement automaticity. J Neurophysiol 92, 2405-2412.

Frey, S.H., 2008. Tool use, communicative gesture and cerebral asymmetries in the modern human brain. Philos Trans R Soc Lond B Biol Sci 363, 1951-1957.

Friston, K., Mattout, J., Trujillo-Barreto, N., Ashburner, J., Penny, W., 2007. Variational free energy and the Laplace approximation. Neuroimage 34, 220-234.

Friston, K.J., Harrison, L., Penny, W., 2003. Dynamic causal modelling. Neuroimage 19, 1273-1302.

Friston, K.J., Williams, S., Howard, R., Frackowiak, R.S., Turner, R., 1996. Movementrelated effects in fMRI time-series. Magn Reson Med 35, 346-355.

Gallivan, J.P., McLean, D.A., Valyear, K.F., Culham, J.C., 2013. Decoding the neural mechanisms of human tool use. Elife 2, e00425.

Garcea, F.E., Mahon, B.Z., 2014. Parcellation of left parietal tool representations by functional connectivity. Neuropsychologia 60, 131-143.

Gharbawie, O.A., Stepniewska, I., Qi, H., Kaas, J.H., 2011. Multiple parietal-frontal pathways mediate grasping in macaque monkeys. J Neurosci 31, 11660-11677.

Ghosh, S., Gattera, R., 1995. A comparison of the ipsilateral cortical projections to the dorsal and ventral subdivisions of the macaque premotor cortex. Somatosens Mot Res 12, 359-378.

Gribova, A., Donchin, O., Bergman, H., Vaadia, E., Cardoso De Oliveira, S., 2002. Timing of bimanual movements in human and non-human primates in relation to neuronal activity in primary motor cortex and supplementary motor area. Exp Brain Res 146, 322-335.

Halsband, U., Lange, R.K., 2006. Motor learning in man: a review of functional and clinical studies. J Physiol Paris 99, 414-424.

Hardwick, R.M., Rottschy, C., Miall, R.C., Eickhoff, S.B., 2013. A quantitative meta-analysis and review of motor learning in the human brain. Neuroimage 67, 283-297.

Hartwigsen, G., Bestmann, S., Ward, N.S., Woerbel, S., Mastroeni, C., Granert, O., Siebner, H.R., 2012. Left dorsal premotor cortex and supramarginal gyrus complement each other during rapid action reprogramming. J Neurosci 32, 16162-16171a.

Jacobs, S., Danielmeier, C., Frey, S.H., 2010. Human anterior intraparietal and ventral premotor cortices support representations of grasping with the hand or a novel tool. J Cogn Neurosci 22, 2594-2608.

Jancke, L., Peters, M., Himmelbach, M., Nosselt, T., Shah, J., Steinmetz, H., 2000. fMRI study of bimanual coordination. Neuropsychologia 38, 164-174.

Jenny, A.B., 1979. Commissural projections of the cortical hand motor area in monkeys. J Comp Neurol 188, 137-145.

Johnson-Frey, S.H., Newman-Norlund, R., Grafton, S.T., 2005. A distributed left hemisphere network active during planning of everyday tool use skills. Cereb Cortex 15, 681-695. 
Karabanov, A., Blom, O., Forsman, L., Ullen, F., 2009. The dorsal auditory pathway is

2 involved in performance of both visual and auditory rhythms. Neuroimage 44, 480-488.

3 Karni, A., Meyer, G., Jezzard, P., Adams, M.M., Turner, R., Ungerleider, L.G., 1995.

4 Functional MRI evidence for adult motor cortex plasticity during motor skill learning.

$5 \quad$ Nature 377, 155-158.

6 Leff, D.R., Leong, J.J., Aggarwal, R., Yang, G.Z., Darzi, A., 2008. Could variations in technical

7 skills acquisition in surgery be explained by differences in cortical plasticity? Ann Surg

$8247,540-543$.

9 Lehericy, S., Benali, H., Van de Moortele, P.F., Pelegrini-Issac, M., Waechter, T., Ugurbil, K.,

10 Doyon, J., 2005. Distinct basal ganglia territories are engaged in early and advanced

11 motor sequence learning. Proc Natl Acad Sci U S A 102, 12566-12571.

Louridas, M., Quinn, L.E., Grantcharov, T.P., 2016. Predictive value of background experiences and visual spatial ability testing on laparoscopic baseline performance among residents entering postgraduate surgical training. Surg Endosc 30, 1126-1133.

Makino, H., Hwang, E.J., Hedrick, N.G., Komiyama, T., 2016. Circuit Mechanisms of Sensorimotor Learning. Neuron 92, 705-721.

Manzoni, T., Barbaresi, P., Conti, F., Fabri, M., 1989. The callosal connections of the primary somatosensory cortex and the neural bases of midline fusion. Exp Brain Res 76, 251-266.

Marangon, M., Kubiak, A., Kroliczak, G., 2015. Haptically Guided Grasping. fMRI Shows Right-Hemisphere Parietal Stimulus Encoding, and Bilateral Dorso-Ventral Parietal Gradients of Object- and Action-Related Processing during Grasp Execution. Front Hum Neurosci 9, 691.

Martin, K., Jacobs, S., Frey, S.H., 2011. Handedness-dependent and -independent cerebral asymmetries in the anterior intraparietal sulcus and ventral premotor cortex during grasp planning. Neuroimage 57, 502-512.

Muakkassa, K.F., Strick, P.L., 1979. Frontal lobe inputs to primate motor cortex: evidence for four somatotopically organized 'premotor' areas. Brain Res 177, 176-182.

Muellbacher, W., Ziemann, U., Wissel, J., Dang, N., Kofler, M., Facchini, S., Boroojerdi, B., Poewe, W., Hallett, M., 2002. Early consolidation in human primary motor cortex. Nature 415, 640-644. Ochiai, T., Mushiake, H., Tanji, J., 2005. Involvement of the ventral premotor cortex in controlling image motion of the hand during performance of a target-capturing task. Cereb Cortex 15, 929-937.

Ohuchida, K., Kenmotsu, H., Yamamoto, A., Sawada, K., Hayami, T., Morooka, K., Takasugi, S., Konishi, K., Ieiri, S., Tanoue, K., Iwamoto, Y., Tanaka, M., Hashizume, M., 2009. The frontal cortex is activated during learning of endoscopic procedures. Surg Endosc 23, 2296-2301.

Palter, V.N., Graafland, M., Schijven, M.P., Grantcharov, T.P., 2012. Designing a proficiency-based, content validated virtual reality curriculum for laparoscopic colorectal surgery: a Delphi approach. Surgery 151, 391-397.

Park, J., Hallett, M., 2015. Investigation of the Transcallosal Ventral Premotor Connection. Neurology 84.

Penhune, V.B., Doyon, J., 2002. Dynamic cortical and subcortical networks in learning and delayed recall of timed motor sequences. J Neurosci 22, 1397-1406.

46 Penhune, V.B., Steele, C.J., 2012. Parallel contributions of cerebellar, striatal and M1 mechanisms to motor sequence learning. Behav Brain Res 226, 579-591.

48 Penny, W.D., Stephan, K.E., Mechelli, A., Friston, K.J., 2004. Comparing dynamic causal models. Neuroimage 22, 1157-1172. 
Pinho, A.L., de Manzano, O., Fransson, P., Eriksson, H., Ullen, F., 2014. Connecting to create: expertise in musical improvisation is associated with increased functional connectivity between premotor and prefrontal areas. J Neurosci 34, 6156-6163.

Puttemans, V., Wenderoth, N., Swinnen, S.P., 2005. Changes in brain activation during the acquisition of a multifrequency bimanual coordination task: from the cognitive stage to advanced levels of automaticity. J Neurosci 25, 4270-4278.

Remy, F., Wenderoth, N., Lipkens, K., Swinnen, S.P., 2008. Acquisition of a new bimanual coordination pattern modulates the cerebral activations elicited by an intrinsic pattern: an fMRI study. Cortex 44, 482-493. Rizzolatti, G., Matelli, M., 2003. Two different streams form the dorsal visual system: anatomy and functions. Exp Brain Res 153, 146-157.

Rouiller, E.M., Babalian, A., Kazennikov, O., Moret, V., Yu, X.H., Wiesendanger, M., 1994. Transcallosal connections of the distal forelimb representations of the primary and supplementary motor cortical areas in macaque monkeys. Exp Brain Res 102, 227-243. Seger, C.A., 2006. The basal ganglia in human learning. Neuroscientist 12, 285-290. Sengul, A., van Elk, M., Rognini, G., Aspell, J.E., Bleuler, H., Blanke, O., 2012. Extending the body to virtual tools using a robotic surgical interface: evidence from the crossmodal congruency task. PLoS One 7, e49473.

Shewokis, P.A., Shariff, F.U., Liu, Y., Ayaz, H., Castellanos, A., Lind, D.S., 2017. Acquisition, retention and transfer of simulated laparoscopic tasks using fNIR and a contextual interference paradigm. Am J Surg 213, 336-345.

Steele, C.J., Penhune, V.B., 2010. Specific increases within global decreases: a functional magnetic resonance imaging investigation of five days of motor sequence learning. J Neurosci 30, 8332-8341.

Stone, K.D., Gonzalez, C.L., 2014. Grasping with the eyes of your hands: hapsis and vision modulate hand preference. Exp Brain Res 232, 385-393.

Stone, K.D., Gonzalez, C.L., 2015. The contributions of vision and haptics to reaching and grasping. Front Psychol 6, 1403.

Townsend, J.T., Ashby, F.G., 1978. Methods of modeling capacity in simple processing systems. In: Castellan, J., Restle, F. (Eds.), Cognitive theory. N.J.: Erlbaum., Hillsdale, pp. 200-239.

Tsuda, H., Aoki, T., Oku, N., Kimura, Y., Hatazawa, J., Kinoshita, H., 2009. Functional brain areas associated with manipulation of a prehensile tool: a PET study. Hum Brain Mapp 30, 2879-2889.

van Eimeren, T., Wolbers, T., Munchau, A., Buchel, C., Weiller, C., Siebner, H.R., 2006. Implementation of visuospatial cues in response selection. Neuroimage 29, 286-294.

van Nuenen, B.F., Kuhtz-Buschbeck, J., Schulz, C., Bloem, B.R., Siebner, H.R., 2012. Weight-specific anticipatory coding of grip force in human dorsal premotor cortex. J Neurosci 32, 5272-5283.

van Polanen, V., Davare, M., 2015. Interactions between dorsal and ventral streams for controlling skilled grasp. Neuropsychologia 79, 186-191.

Veale, J.F., 2014. Edinburgh Handedness Inventory - Short Form: a revised version based on confirmatory factor analysis. Laterality 19, 164-177.

Ward, N.S., Bestmann, S., Hartwigsen, G., Weiss, M.M., Christensen, L.O., Frackowiak, R.S., Rothwell, J.C., Siebner, H.R., 2010. Low-frequency transcranial magnetic stimulation over left dorsal premotor cortex improves the dynamic control of visuospatially cued actions. J Neurosci 30, 9216-9223.

Woodrum, D.T., Andreatta, P.B., Yellamanchilli, R.K., Feryus, L., Gauger, P.G., Minter, R.M., 2006. Construct validity of the LapSim laparoscopic surgical simulator. Am J Surg 191, 28-32. 
1 Wymbs, N.F., Bassett, D.S., Mucha, P.J., Porter, M.A., Grafton, S.T., 2012. Differential 2 recruitment of the sensorimotor putamen and frontoparietal cortex during motor 3 chunking in humans. Neuron 74, 936-946.

4 Zhu, F.F., Poolton, J.M., Wilson, M.R., Hu, Y., Maxwell, J.P., Masters, R.S., 2011. Implicit 5 motor learning promotes neural efficiency during laparoscopy. Surg Endosc 25, 295062955. 
Figure 1: Experimental procedures. A) Endoscopic setup inside the scanner: A1 displays the participant lying on the scanner bed with the Endoscopic box trainer and instruments in place before the start of the experiment. A2 shows the same participant after having reached the final experimental position inside the scanning bore. A3 illustrates the endoscopic workspace seen from the top and A4 the endoscopic workspace seen from the participants' perspective via a camera placed inside the endoscopy box. In this image the participant can be seen performing the 'endoscopic Transfer' task. B) Endoscopic setup for VR-training on an endoscopic simulator. B1 shows a participant training on the simulator. B2 displays a simple training task, similar to the in-scanner exercise and B3 displays a more complex training task, simulating endoscopic vessel cutting. C) Timeline of the experimental design. The training, displayed in $1 \mathrm{~B}$, is represented by the grey-white box, with the dashed line indicating that only the 'Training Group' $(n=24)$ took part in these sessions.

Figure 2: Overall endoscopic performance in the training setting. Group data are given as mean and standard deviation of mean showing the relative mean improvement across all tasks over the 5-day VR-training relative to baseline performance for the training group. To compute an overall mean performance score each individual score was normalized such that values lay between 0 and 1. The data obtained at day 2-5 are calculated by subtracting the individual performance value on training day 1 from the performance values recorded on subsequent days.

Figure 3: Endoscopic performance within the MRI scanner. Group data are given as mean and standard deviation. A) Performance speed during the 'Simple Pointing' fMRI task reflected by the average number of completed 'pointing cycles' (e.g. left-right pointing) within one $40 \mathrm{~s}$ task block. B) Performance speed for the complex 'Endoscopic Transfer' fMRI task during a $40 \mathrm{~s}$ task block indicated as number of completed 'transfer cycles' (e.g. Pick up - Transfer - Place down). C) Accuracy during the complex task reflected by the average number of the ring being dropped in a $40 \mathrm{~s}$ task block. For all measures, the data obtained during the second and third fMRI session at day 2 and 5 are calculated by subtracting the individual performance value during the first fMRI session at day 1 from the performance values recorded at day 2 and 5 .

Figure 4: A) Colour-coded statistical parametric maps (SPMs) showing group-independent effect of performing the complex endoscopic task relative to the pointing task during the baseline scanning session. The colour coding reflects the voxel-specific Z-score and the cluster extent of the SPMs are thresholded at FWE $=0.05$ voxel-level. B) SPMs displaying the group-independent increase in left putamen. In the right PMd and bilaterally in the border zone between left PMv and IFG during the complex 'Endoscopic Transfer' task, occurring from the first to the second scanning session. C) SPMs showing the group-independent increase at the border of left PMv and IFG and in the left aIPS during the complex 'Endoscopic Transfer' task observed from the first to the third scanning session. Yellow colours activations indicate that SPMs were thresholded at FWE = 0.05 voxel-level, orange colour coding indicate at a threshold of FWE $=0.05$ small volume corrected for the grasping network (Binkofski et al., 1999). The activation maps are superimposed on a SPM template image (avg305T1.img) 
1 Figure 5: Significant increases in task-related activity (bilateral transfer minus simple pointing) over time in the 2 training group only (red) and with a significant increase over time in both groups (yellow). The statistical 3 activation maps on the left are FWE-corrected at $\mathrm{p}<0.05$ and bar graphs show the relative (\%) signal change in 4 the left and right PMv and IFG region respectively (bilateral transfer minus simple pointing). The maps 5 presented on the right have been FEW-corrected for small volume at $\mathrm{p}<0.05$ (see text for details). Darker 6 colouring (dark red and orange) indicates that an area was significant at a threshold of FWE $>0.05$ small volume 7 correction for the grasping network.

9 Figure 6: Effective connectivity analysis using DCM. A) The left panel gives the selected model parameters, including the A- and B-matrixes. B) Panel B gives the results of Bayesian model comparison indicating the

11 winning models for each of the two experimental groups. C) Illustration of the modulatory effect of time on the left-to-right PMv connection in the training group as revealed by DCM.

14 Figure 7: Cluster in left M1-HAND showing a positive linear relationship between task-related activation for bimanual transfer (relative to pointing) and individual improvement in endoscopic skills over the entire training period. A) Relative performance improvement during VR-training in the training group (mean change from baseline \pm SD). B) SPM of voxels showing a linear positive relationship between change in task-related activity within left M1-HAND and individual training benefit (small volume corrected FWE at $p<0.05$ ). C)

19 Corresponding scatter plot and regression line (blue). 


\section{ACCEPTED MANUSCRIPT}

Table 1. Sample demographic

N Women (\%) Age

Right

Semester

Interested in Musical

$M(S D)$

handedness Medicine

surgical career instrument

(\%)

$\mathrm{M}(\mathrm{SD})$

(\%)

(\%)

\begin{tabular}{llllllll}
\hline fMRI & & & & & & \\
Training group & 24 & 41.67 & $24.00(1.87)$ & 94.58 & $6.67(3.15)$ & 29.16 & 41.67 \\
Control group & 24 & 58.33 & $22.62(2.12)$ & 85.20 & $5.58(2.94)$ & 37.50 & 16.67 \\
\hline$M(S D)$ - Mean (Standard deviation) & &
\end{tabular}

$M(S D)$ - Mean (Standard deviation) 
Table 2: Complex Task activations during the first fMRI session (baseline scan). Correction for multiple comparisons used the FWE method at the voxel level applying a corrected $\mathrm{p}<0.05$

\begin{tabular}{|c|c|c|c|c|c|c|}
\hline \multirow[t]{2}{*}{ Region } & \multirow[t]{2}{*}{ BA } & \multicolumn{3}{|c|}{$\begin{array}{l}\text { MNI } \\
\text { coordinates }\end{array}$} & \multirow{2}{*}{\multicolumn{2}{|c|}{$\begin{array}{l}\text { Cluster } \\
\text { Size }\end{array}$}} \\
\hline & & $\boldsymbol{x}$ & $y$ & $Z$ & & \\
\hline SPL L & $\begin{array}{l}7 \mathrm{a} \\
7 \mathrm{p}\end{array}$ & $\begin{array}{l}-15 \\
-27\end{array}$ & $\begin{array}{l}-70 \\
-59\end{array}$ & $\begin{array}{l}53 \\
64\end{array}$ & 413 & 8.92 \\
\hline $\begin{array}{l}\text { SPL R } \\
\text { Fusiform G L }\end{array}$ & $\begin{array}{l}7 \mathrm{a} \\
7 \mathrm{P}\end{array}$ & $\begin{array}{l}18 \\
21\end{array}$ & $\begin{array}{l}-67 \\
-61\end{array}$ & $\begin{array}{l}53 \\
59\end{array}$ & 383 & 8.20 \\
\hline $\begin{array}{l}\text { Inf. Occipital G R } \\
\text { Lateral Occipital G L }\end{array}$ & 18 & $\begin{array}{c}39 \\
51 \\
39\end{array}$ & $\begin{array}{c}85 \\
61 \\
92\end{array}$ & $\begin{array}{r}2 \\
16 \\
11\end{array}$ & 354 & 7.93 \\
\hline $\begin{array}{l}\text { Inf. Occipital G L } \\
\text { Lateral Occipital G L }\end{array}$ & 18 & $\begin{array}{l}-36 \\
-51 \\
-45\end{array}$ & $\begin{array}{r}88 \\
-73 \\
73\end{array}$ & $\begin{array}{c}11 \\
-4 \\
19\end{array}$ & 413 & 7.63 \\
\hline $\begin{array}{l}\text { Cb. Vermis } 7 \\
\text { Cb. Vermis } 10 \\
\text { Lobule VIII }\end{array}$ & & $\begin{array}{l}3 \\
0 \\
0\end{array}$ & $\begin{array}{l}-76 \\
-52 \\
-73\end{array}$ & $\begin{array}{l}-19 \\
-22 \\
-37\end{array}$ & 354 & 7.18 \\
\hline Thalamus & & $\begin{array}{c}0 \\
-9 \\
12\end{array}$ & $\begin{array}{r}-25 \\
-16 \\
31\end{array}$ & $\begin{array}{r}-4 \\
-4 \\
3\end{array}$ & 87 & 6.94 \\
\hline Premotor $\mathbf{R}$ & 6 & 33 & 7 & 71 & 58 & 6.42 \\
\hline Cb. VIII L & & -24 & -43 & -43 & 36 & 5.56 \\
\hline S1/M1 R & $\begin{array}{l}3 a \\
1\end{array}$ & $\begin{array}{l}63 \\
57\end{array}$ & $\begin{array}{l}-16 \\
-19\end{array}$ & $\begin{array}{l}41 \\
50\end{array}$ & 23 & 5.88 \\
\hline Premotor L & 6 & -24 & -1 & 71 & 16 & 5.06 \\
\hline S1/M1 L & 2 & 45 & -37 & 56 & 12 & 5.04 \\
\hline
\end{tabular}




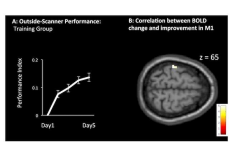

I:

? 


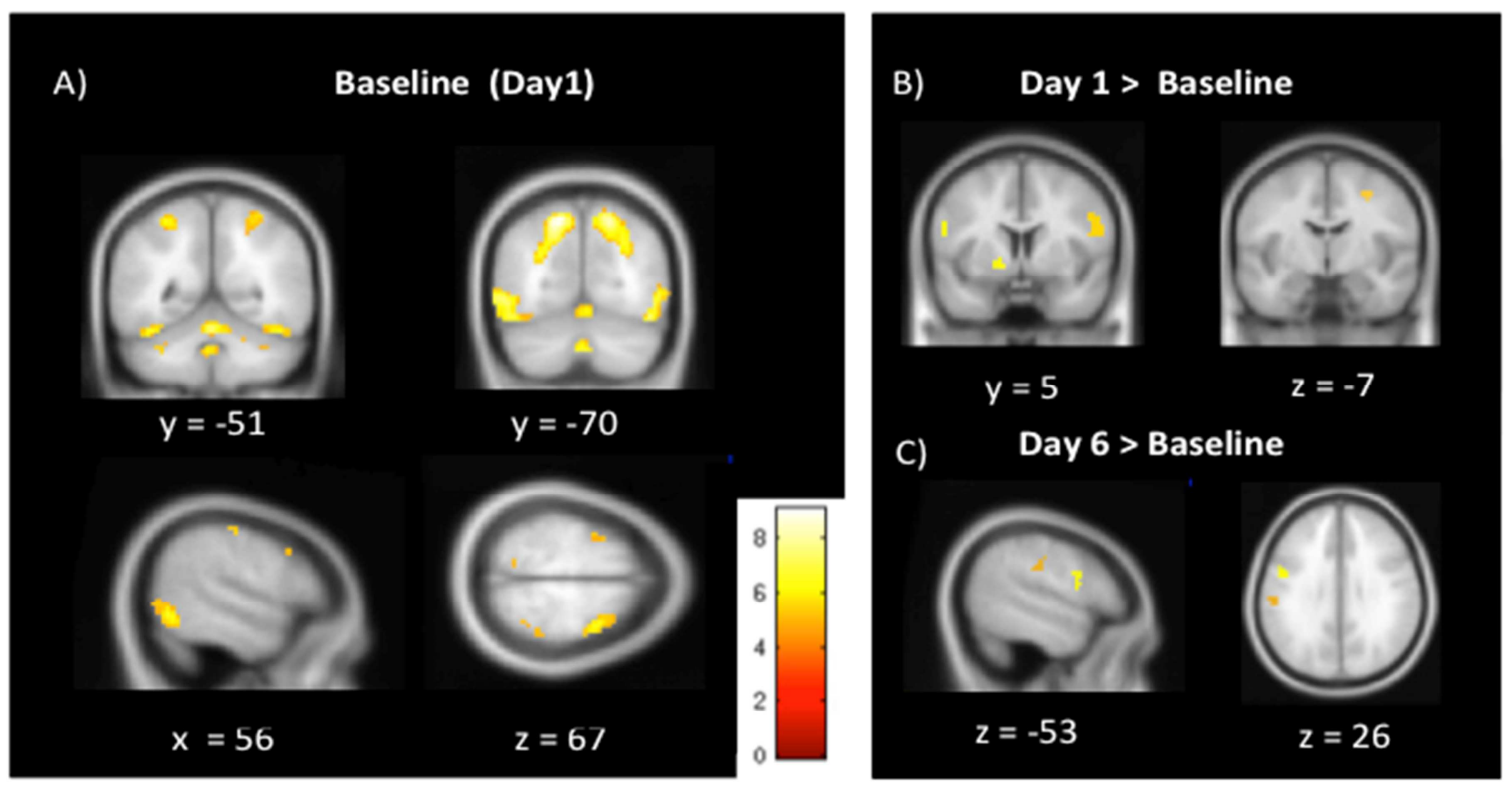


Outside-Scanner Performance: Training Group

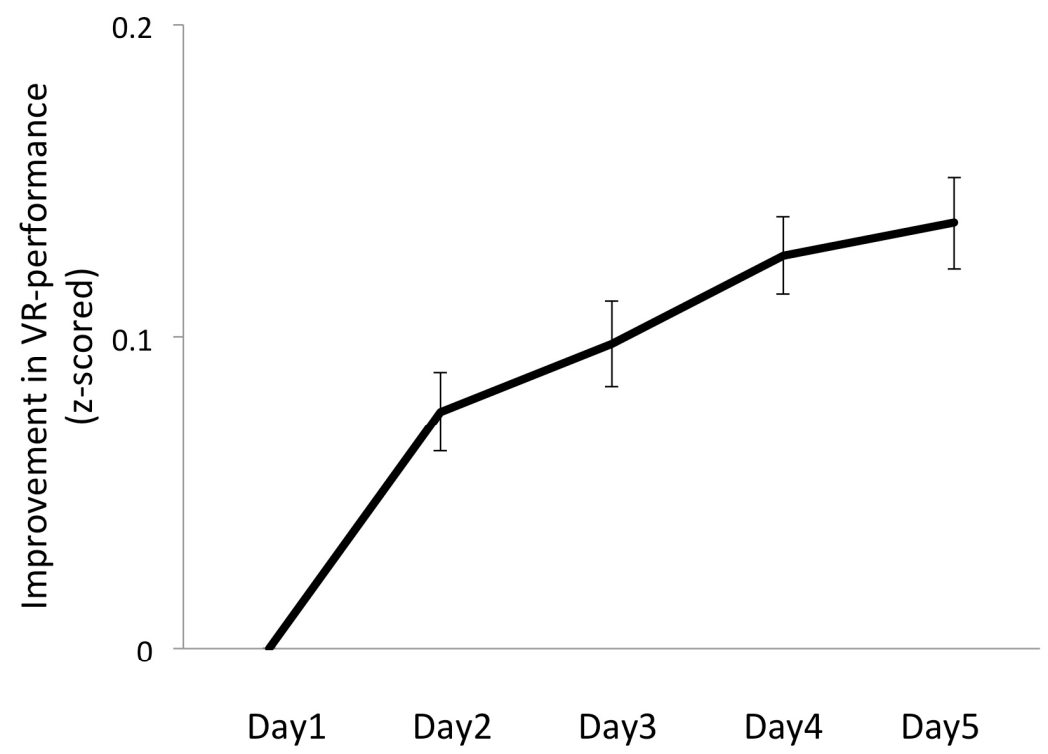


A) Within Scanner Performance:

Pointing Speed

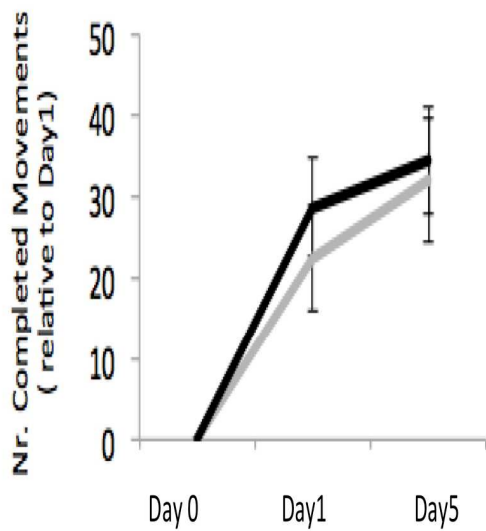

B) Within Scanner Performance:

Transfer Speed

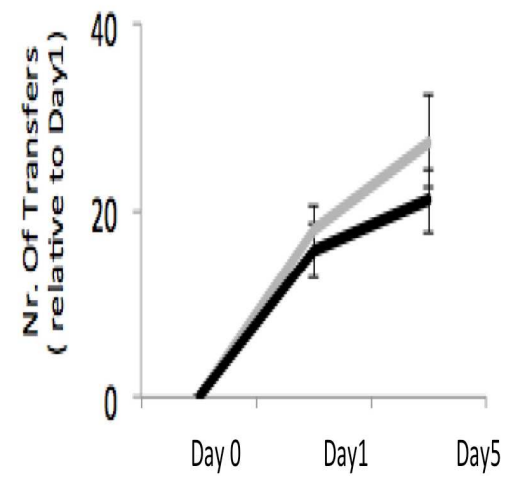

C) Within Scanner Performance: Error Number

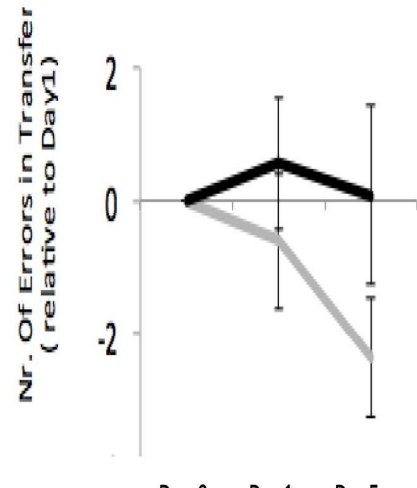

Day0 Day1 Day5

- Training Group $=$ Control Group 
A) Model Selection
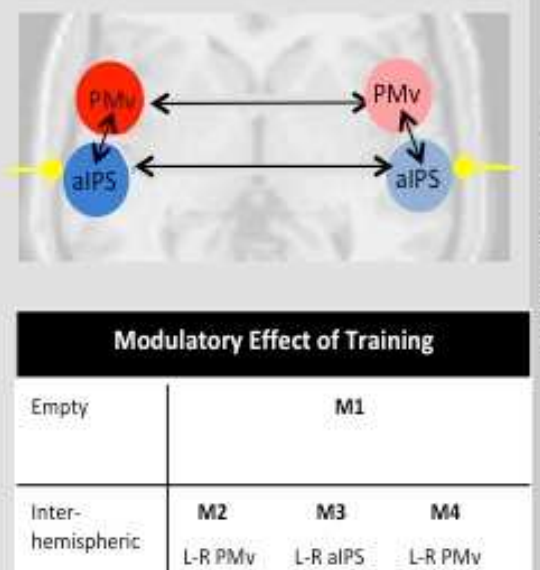

\begin{tabular}{|c|c|c|}
\hline hemispheric & L-R PMv L-R alPS & $\begin{array}{l}\text { L-R PMv } \\
\text { L-R aiPS }\end{array}$ \\
\hline Intra & M6 & M7 \\
\hline hemispheric & LPMv-aIPS R PMv-alPS & $\begin{array}{l}\text { LPMv-alPS } \\
\text { RPMv-alPS }\end{array}$ \\
\hline \multirow{2}{*}{$\begin{array}{l}\text { Intra / Inter } \\
\text { hernispheric }\end{array}$} & \multicolumn{2}{|l|}{ M8 } \\
\hline & \multicolumn{2}{|c|}{$\begin{array}{ll}\text { L-RPMv } & \text { LPMv-alPS } \\
\text { L-R aIPS } & \text { RPMv-alPS }\end{array}$} \\
\hline
\end{tabular}

B) Model Comparison

Training Group

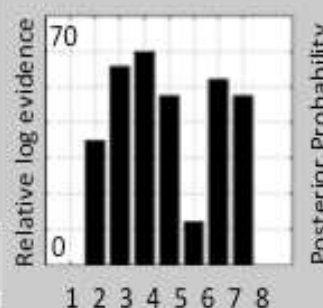

12345678

Control Group

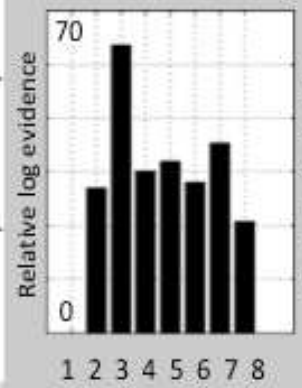

C) Parameter Comparison

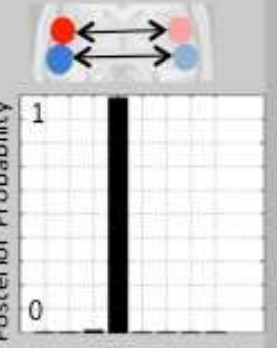

12345678
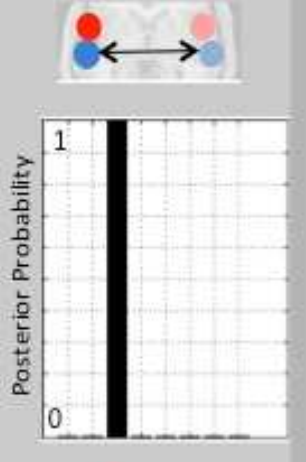

12345678
Training Group

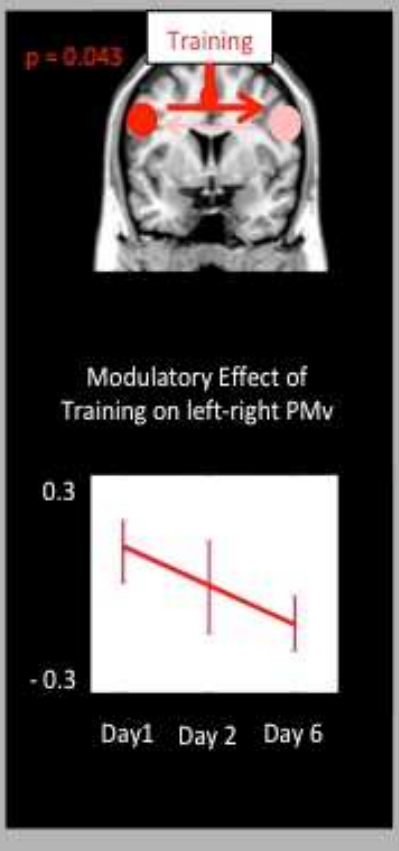


A) Within-Scanner Setup
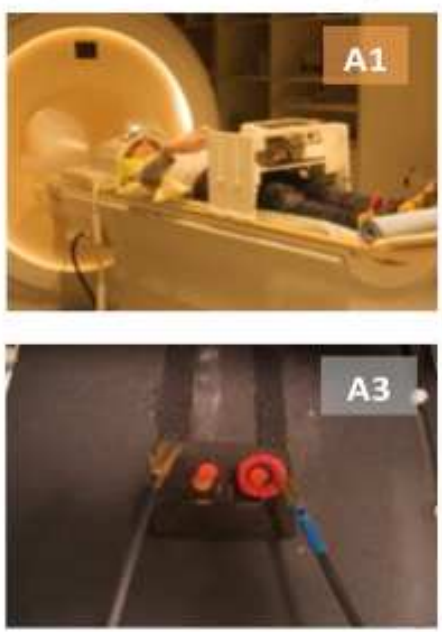

C) Experimental Design

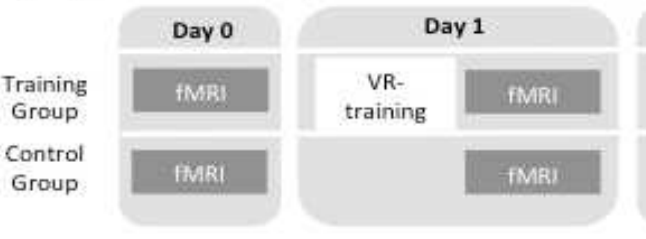

B) Outside -Scanner Setup
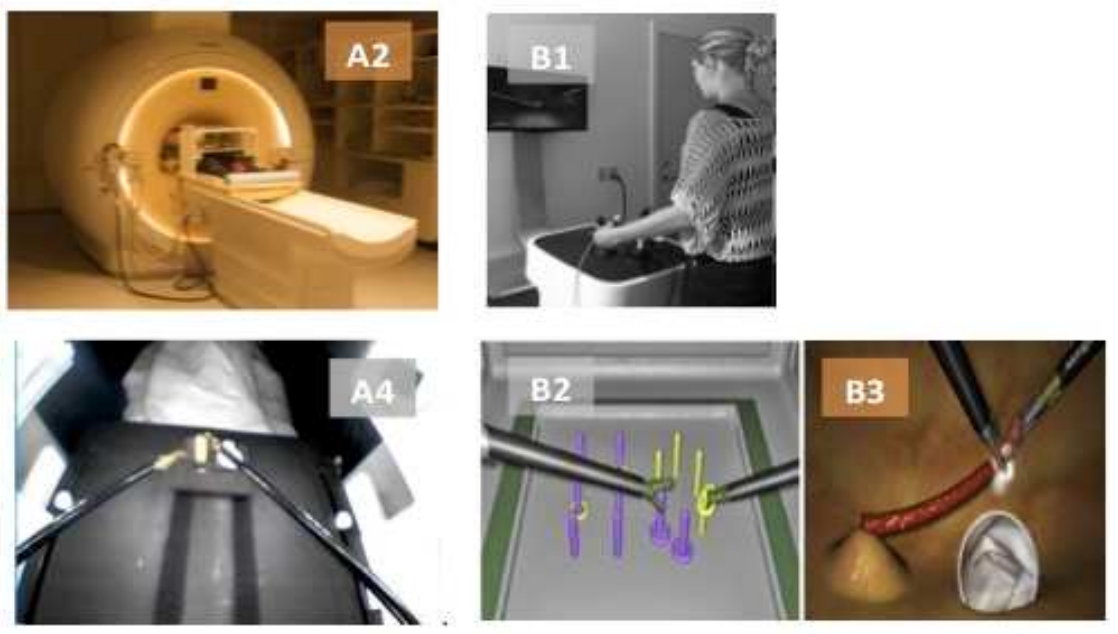

VR-
training

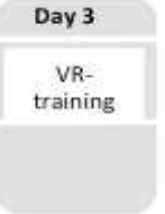

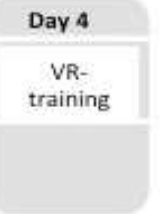

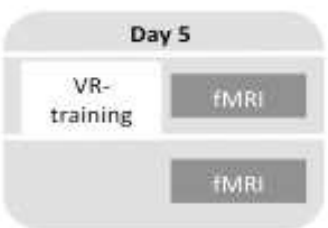




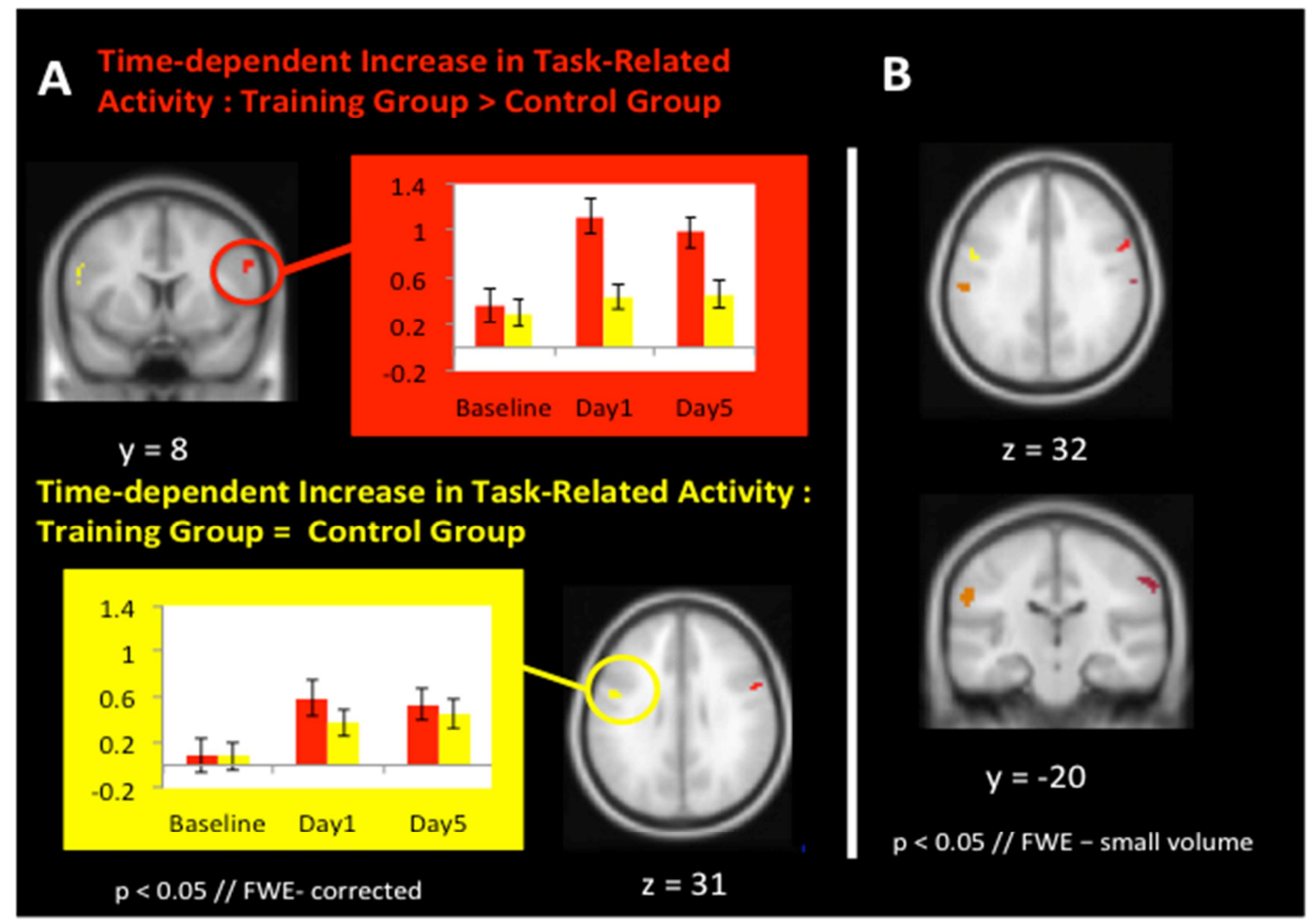

